\title{
A SIMULINK Block Set for the High-Level Simulation of Multistandard Radio Receivers
}

\author{
Alonso Morgado, Rocío del Río and José M. de la Rosa \\ Instituto de Microelectrónica de Sevilla - IMSE-CNM (CSIC) \\ Edif. CICA-CNM, Avda. Reina Mercedes s/n, 41012- Sevilla, SPAIN \\ Phone: +34 95 5056666, Fax: +34 95 5056686, E-mail: \{alonso|rocio|jrosa\}@imse.cnm.es
}

\begin{abstract}
This paper describes a SIMULINK block set for the behavioral simulation of $\mathrm{RF}$ receivers. Building blocks are modeled including their main circuit-level non idealities. These models are incorporated into the SIMULINK environment making an extensive use of $\mathrm{C}$-coded $\mathrm{S}$-functions and reducing the number of library block elements. This approach reduces the simulation time while keeping high accuracy, what makes the proposed toolbox very appropriate to be combined with an optimizer for the automated high-level synthesis of radio receivers. As a case study, a direct-conversion receiver intended for $4 \mathrm{G}$ telecom systems is modeled and simulated using the proposed toolbox. ${ }^{\dagger 1}$
\end{abstract}

\section{INTRODUCTION}

It is expected that with the so-called fourth generation (4G) of mobile telecommunications, different access systems such as cellular, satellite, WLAN-based and short-range connectivity will be combined on a common platform to complement each other in an optimal way [1]. These new telecom systems will require multistandard RF transceivers, highly integrated in very low-cost deep-submicron CMOS technologies, and capable of being reconfigured to operate according to as many as possible communication standards with the minimum power consumption [2][3].

In order to achieve these objectives, proper CAD tools and design methodologies are needed to optimize the system planning and to reduce the time-to-market deployment [4][5]. Although a number of commercial and academic tools for the design and verification of RF circuits and systems are known [6][7][8], very few has been done in the field of automated synthesis methodology - from specifications to silicon [3]. In addition, a systematic exploration of different alternative implementations, in terms of architecture selection, reconfiguration strategies and circuit-level implementation is needed in order to optimize multistandard transceivers.

This paper contributes to this topic and focuses on the behavioral modeling of RF transceivers for multistandard telecommunication systems in the MATLAB/SIMULINK environment [9]. This platform provides a number of advantages: (a) it is a widely used platform, familiar to a large number of engineers; (b) it has direct access to very powerful tools for signal processing and data manipulation; (c) it has complete flexibility to create new transceiver architectures, and even to include different blocks; and (d) it enables a high flexibility for the

$\dagger 1$. This work has been supported by the Spanish Ministry of Science and Education (with support from the European Regional Development Fund) under contract TEC2004-01752/MIC extension of the block library and adding of new blocks or models without requiring the qualified contribution of a programmer. The toolbox includes a complete list of RF circuit models that take into account their main circuit limitations. These models are implemented by properly combining elementary SIMULINK library blocks with S-functions in order to decrease the computational cost [9]. As an application, a direct-conversion receiver for GSM/UMTS/Bluetooth/WLAN is modeled based on the presented approach.

\section{PROPOSED SIMULINK RF BLOCK SET}

The presented toolbox includes a library of RF building blocks that are needed to implement RF receiver front-ends, namely: Low Noise Amplifiers (LNAs), mixers, oscillators, fil-

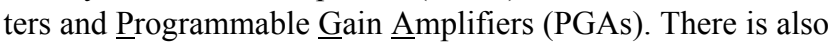
a library including other blocks like the antenna, duplexer filter and antenna switches, required to implement reconfigurable architectures. Behavioral models of building blocks include the main ideal functionality as well as the following non-idealities:

- Thermal noise, characterized by the Noise Figure $(N F)$ and

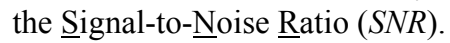

- Nonlinearity, commonly expressed by the input-referred 2nd- and 3rd-order intercept points, $I I P_{2}$ and $I I P_{3}$, respectively [10].

In addition to these general parameters, some block specific errors have been also included, like for instance, oscillator phase noise and mixer offset [10]. All these models have been incorporated in the proposed SIMULINK toolbox as described below.

\section{A. Amplifiers}

There are two kinds of amplifiers included in the toolbox: LNAs and PGAs. Both of them are modeled by the block diagram shown in Fig.1. It consists of a S-function block that includes the non-linear characteristic of the amplifier followed by a Gaussian noise source which is added at the output node

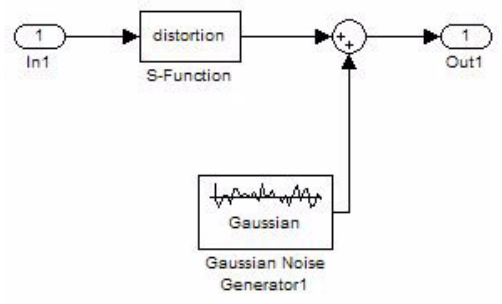

Figure 1. Model block diagram of LNAs and PGAs. 
of the block. The non-linear function is modeled as:

$$
v_{o}=v_{o f f}+A_{V} v_{i n}\left(1+\frac{v_{i n}}{\left|\rho_{i 2}\right|}+\frac{4}{3} \frac{v_{i n}^{2}}{\rho_{i 3}^{2}}\right)
$$

where $v_{\text {off }}$ stands for the offset voltage, $A_{V}$ is the voltage gain, which can be programmable in the case of PGAs, and $\rho_{i 2}$ and $\rho_{i 3}$ are the second- and third-order intercept points, respectively.

Note that the model in (1) can be easily implemented by using SIMULINK elementary library blocks as shown in Fig. 2. However, this causes the MATLAB interpreter to be called at each time step, slowing down the simulation time drastically. This problem is aggravated as the circuit complexity increases, yielding to excessive CPU times which can be prohibitive to use these models as an evaluation vehicle in an optimization loop. This is true even using the SIMULINK accelerator [9].

In order to avoid this problem, the non-linear characteristic has been codified using C-compiled S-functions. For this purpose, SIMULINK provides different $\mathrm{S}$-function templates which can accommodate the $\mathrm{C}$-coded computation model of different systems. These templates are composed of several routines that perform different tasks required at each simulation stage. Once the S-function has been created, it is compiled by using the mex utility provided by MATLAB and the resulting object files are dynamically linked into SIMULINK when needed [9].

\section{B. Mixers}

Fig. 3 shows the mixer model included in the toolbox. In addition to the main operation, the model incorporates a non-linear block and an additive thermal noise source in a similar way to the amplifier model. Note that there is an additional gain path, $f_{\mathrm{db}}$, that is used to model the so-called direct

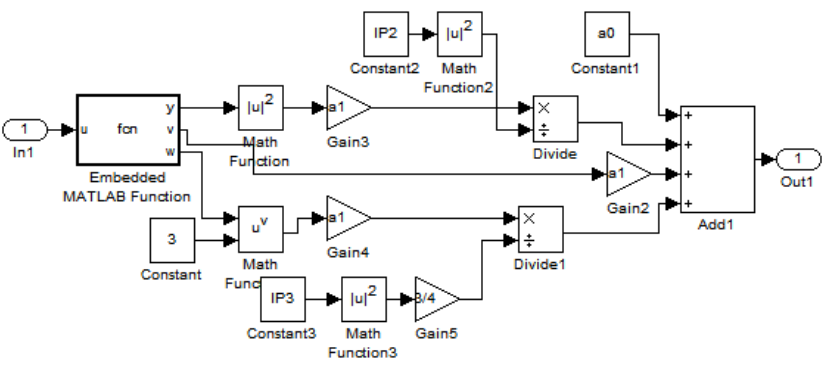

Figure 2. Implementation of (1) using SIMULINK library blocks.

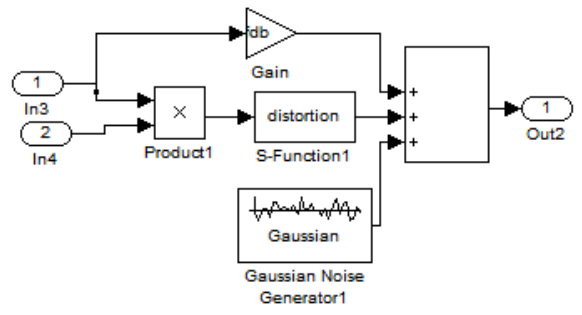

Figure 3. Model block diagram of mixers. feedthrough effect of RF components caused by mismatch-induced asymmetries in the mixer [10].

\section{Filters}

The model used for filters, shown in Fig.4, is very similar to that used for the amplifiers and mixers except for the filtering function itself - implemented by a SIMULINK library element. In addition, a voltage gain is used to compensate for filter attenuation. Note that different kind of filter functions, either lowpass (for baseband) or bandpass (for RF) are considered as well as main synthesis methods, namely: Butterworth, Chebyshev, Cauer and Bessel.

\section{Oscillators}

Quadrature oscillators have been incorporated in the toolbox. As illustrated in Fig.5(a), the model consists of two sinewave (In-phase and Quadrature) signals of amplitude $1 \mathrm{~V}$ and $90^{\circ}$ phase shift. There are two important errors included in the model: I/Q imbalance and phase noise. The former is modeled as shown in Fig.5(b), by including a phase and amplitude mismatch implemented with SIMULINK elementary blocks.

Phase noise has been modeled by using SIMULINK library blocks as illustrated in Fig.5(c), in which an additive noise source is generated, digitally filtered and added to the phase of the original signal, $x$, giving [10]:

$$
x(t)=A \cos \left(\omega_{c} t+\phi_{n}(t)\right) \cong A \cos \omega_{c} t-A \phi_{n}(t)
$$

where $A$ and $\omega_{c}$ are the amplitude and frequency of the signal, respectively, and $\phi_{n}$ stands for the phase noise.

\section{E. Antenna and switch}

The model used for the antenna includes an additive thermal noise source and the test signal. The latter is different depending on the test applied to verify the corresponding standard figures, as will be shown in next sections. In the case of multistandard receivers, a switch block is required to model the connection of separate RF paths, one per standard. This block is modeled as a non-linear block in a similar way to amplifiers, mixers and filters.

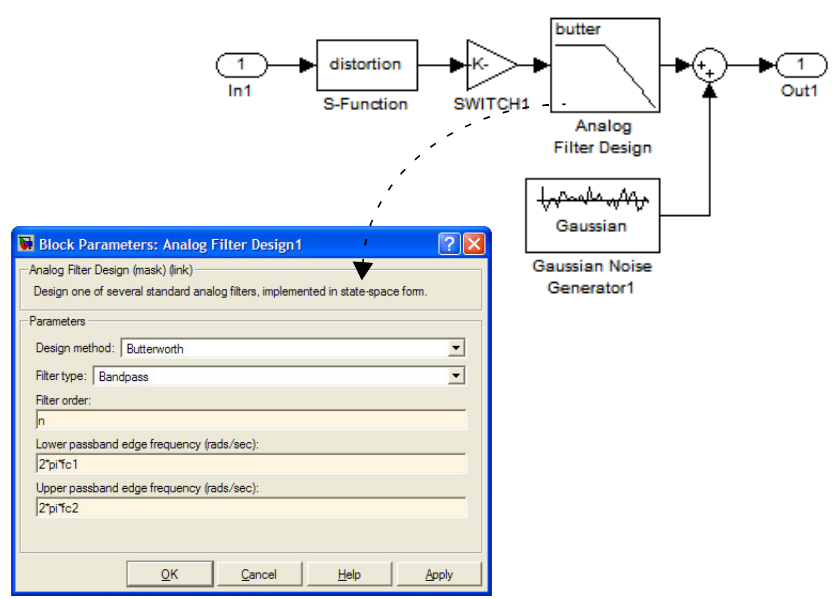

Figure 4. Model block diagram of filters. 


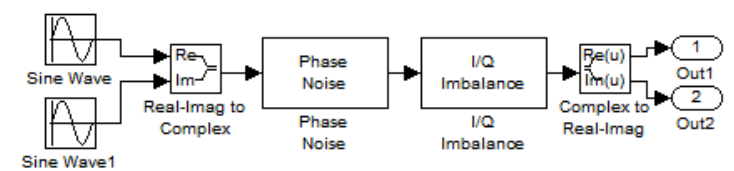

(a)

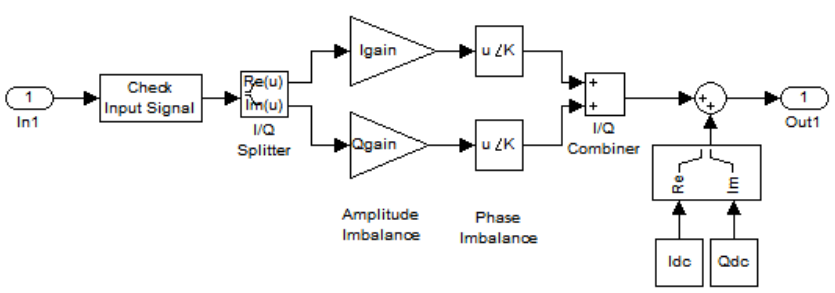

(b)

dc
Offset

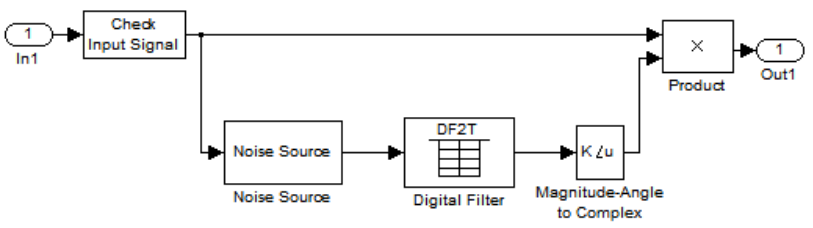

(c)

Figure 5. Modeling oscillators. (a) Model block diagram. (b) I/Q imbalance model. (c) Phase Noise model.

\section{APPLICATION TO MULTISTANDARD RECEIVERS}

As an application of the capabilities of the presented toolbox, a multistandard Direct-Conversion Receiver (DCR) has been modeled using the building blocks described in previous sections. The corresponding block diagram in SIMULINK is shown in Fig.6. This receiver architecture is commonly used in multistandard applications because it eliminates the need for both IF and image rejection filtering and requires only a single oscillator and mixer [11]. In order to cope with the requirements of the different standards, separate (switchable) RF hardware paths (normally one per standard) are used whereas a single, digitally-programmed baseband section (from the mixer to the ADC) is implemented [12]. It is important to mention that the receiver includes the signal processing from the antenna to the Analog-to-Digital Converter (ADC). However, this block is implemented by simply including a non-linear function and additive noise similarly to an unity-gain amplifier [13].

In this example, the multistandard receiver in Fig. 6 must fulfill the performance requirements of four standards: GSM,
Bluetooth, UMTS and WLAN, summarized in Table I. These requirements are normally mapped onto building-block specifications (gain, dynamic range, linearity, and noise figure) in an iterative synthesis process, generally referred to as receiver planning [3].

A complete receiver planning - beyond the scope of this paper - can be done by combining the proposed toolbox with an optimizer, in which every building-block specification is a design parameter that has to be found out for given specifications. Another application of the toolbox might be to find out the specifications of one building block, assuming fixed specifications for the remaining blocks in the receiver. As an illustration of this process, the ADC effective resolution was obtained considering that the specifications of the other blocks in the receiver chain were those given in Table II - extracted from previously reported designs $[13]^{\dagger 2}$. For that purpose, an iterative simulation-based procedure was done considering the

TABLE I. INPUT-REFERRED REQUIREMENTS FOR EACH STANDARD

\begin{tabular}{|l|c|c|c|c|}
\cline { 2 - 5 } \multicolumn{1}{c|}{} & GSM & Bluetooth & UMTS & WLAN \\
\hline Sensitivity & $-102 \mathrm{dBm}$ & $-70 \mathrm{dBm}$ & $-117 \mathrm{dBm}$ & $-65 \mathrm{dBm}$ \\
\hline Max. signal & $-15 \mathrm{dBm}$ & $-20 \mathrm{dBm}$ & $-25 \mathrm{dBm}$ & $-30 \mathrm{dBm}$ \\
\hline Bandwidth & $200 \mathrm{kHz}$ & $1 \mathrm{MHz}$ & $3.84 \mathrm{MHz}$ & $20 \mathrm{MHz}$ \\
\hline Interferer level & $-49 \mathrm{dBm}$ & $-39 \mathrm{dBm}$ & $-46 \mathrm{dBm}$ & $-45 \mathrm{dBm}$ \\
\hline Max. out-band blocker & $0 \mathrm{dBm}$ & $-10 \mathrm{dBm}$ & $-15 \mathrm{dBm}$ & $0 \mathrm{dBm}$ \\
\hline Max. in-band blocker & $-23 \mathrm{dBm}$ & --- & $-44 \mathrm{dBm}$ & $-30 \mathrm{dBm}$ \\
\hline Max. adjacent channel & $-33 \mathrm{dBm}$ & $-27 \mathrm{dBm}$ & $-92.7 \mathrm{dBm}$ & $-65 \mathrm{dBm}$ \\
\hline
\end{tabular}

TABLE II. RECEIVER BUILDING-BLOCK SPECIFICATIONS

\begin{tabular}{|c|c|c|c|c|c|c|c|c|}
\hline & \multirow{2}{*}{\multicolumn{2}{|c|}{ SWITCH }} & \multirow{2}{*}{\multicolumn{2}{|c|}{ RF FILTER }} & \multicolumn{4}{|c|}{ LNA } \\
\hline & & & & & \multicolumn{2}{|c|}{$G_{\max .}$} & \multicolumn{2}{|c|}{$G_{\min .}$} \\
\hline Gain (dB) & \multicolumn{2}{|c|}{-2} & \multicolumn{2}{|c|}{-1} & \multicolumn{2}{|c|}{18} & \multicolumn{2}{|c|}{8} \\
\hline$N F(\mathrm{~dB})$ & \multicolumn{2}{|c|}{0} & \multicolumn{2}{|c|}{0} & \multicolumn{2}{|c|}{3.5} & \multicolumn{2}{|c|}{5.0} \\
\hline$I I P_{3}(\mathrm{dBm})$ & \multicolumn{2}{|c|}{50} & \multicolumn{2}{|c|}{50} & \multicolumn{2}{|c|}{-5} & \multicolumn{2}{|c|}{0} \\
\hline \multirow[t]{2}{*}{$I P_{2}(\mathrm{dBm})$} & \multicolumn{2}{|c|}{80} & \multicolumn{2}{|c|}{80} & \multicolumn{2}{|c|}{25} & \multicolumn{2}{|c|}{30} \\
\hline & \multicolumn{4}{|c|}{ MIXER } & \multicolumn{4}{|c|}{ LPF } \\
\hline Gain (dB) & \multicolumn{4}{|c|}{2} & \multicolumn{4}{|c|}{0} \\
\hline$N F(\mathrm{~dB})$ & \multicolumn{4}{|c|}{20} & \multicolumn{4}{|c|}{25} \\
\hline$I I P_{3}(\mathrm{dBm})$ & \multicolumn{4}{|c|}{8} & \multicolumn{4}{|c|}{8} \\
\hline$I I P_{2}(\mathrm{dBm})$ & \multicolumn{4}{|c|}{55} & \multicolumn{4}{|c|}{65} \\
\hline \multirow[t]{4}{*}{ Order } & \multicolumn{4}{|c|}{---} & \multicolumn{4}{|c|}{6} \\
\hline & & & & & & & & \\
\hline & GS & SM & & & & & & $\mathbf{A N}$ \\
\hline & $\mathrm{S}_{\min }$ & $\mathrm{S}_{\max }$ & $\mathrm{S}_{\min }$ & $\mathrm{S}_{\max }$ & $S_{\min }$ & $S_{\max }$ & $S_{\min }$ & $\mathrm{S}_{\max }$ \\
\hline Gain (dB) & 97 & 20 & 66 & 24 & 112 & 30 & 66 & 24 \\
\hline$N F(\mathrm{~dB})$ & 10 & 25 & 10 & 25 & 10 & 25 & 10 & 25 \\
\hline$I I P_{3}(\mathrm{dBm})$ & -40 & 8 & -40 & 8 & -40 & 8 & -34 & 8 \\
\hline$I I P_{2}(\mathrm{dBm})$ & 10 & 65 & 20 & 60 & 10 & 65 & 10 & 65 \\
\hline
\end{tabular}

$\dagger 2$. In the case of GSM and UMTS, extrapolated data from [13] were taken.

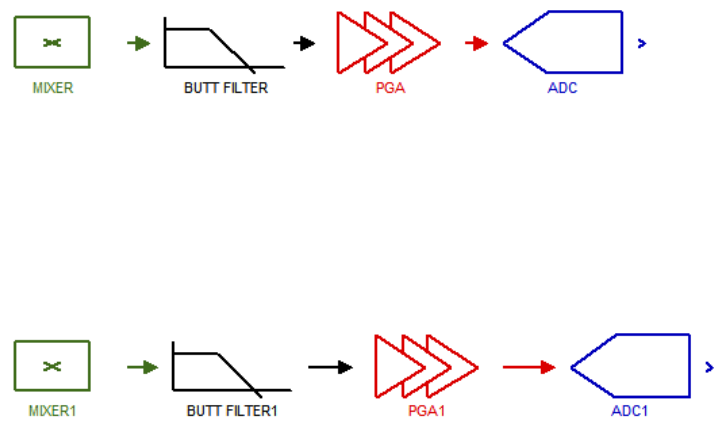

Figure 6. Model block diagram of the multistandard DCR. 


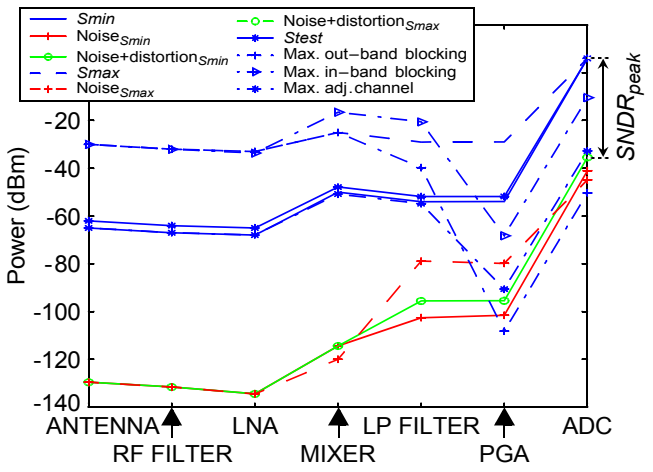

Figure 7. Propagation of different performance metrics for WLAN.

propagation of the different standard test signals through the receiver front-end. Fig. 7 depicts the propagation of the maximum and minimum signal (sensitivity) levels (Smax and Smin, respectively) from the antenna to the ADC input, together with that of noise and distortion for the WLAN stand-

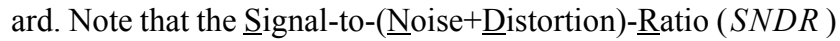
peak at the ADC input is measured as the difference of Smax to the noise plus distortion. The test recommended by the standard with maximum spurious signals is also included, with Stest being the wanted signal level.

\section{SIMULATION RESULTS}

The DCR in Fig. 6 was simulated considering the building-blocks specifications shown in Table II. Different test signals and interferers (in-band blockers, out-band blockers, adjacent channel signals, etc.) were applied in order to verify the receiver performance according to the standard specifications. As an illustration, Fig. 8 depicts the level diagram of these signals when they are propagated through the receiver chain for the UMTS case.

The main performance metrics of RF receivers are $N F, I I P_{2}$ and $I I P_{3}$. The contribution of each building block to the overall value of these figures can be evaluated by using the proposed toolbox. This is illustrated in Fig.9 where the evolution of $N F$ and $I I P_{3}$ through the receiver is represented for each standard. Finally, Table III sums up the simulated performance of the receiver which is in good agreement with specifications.

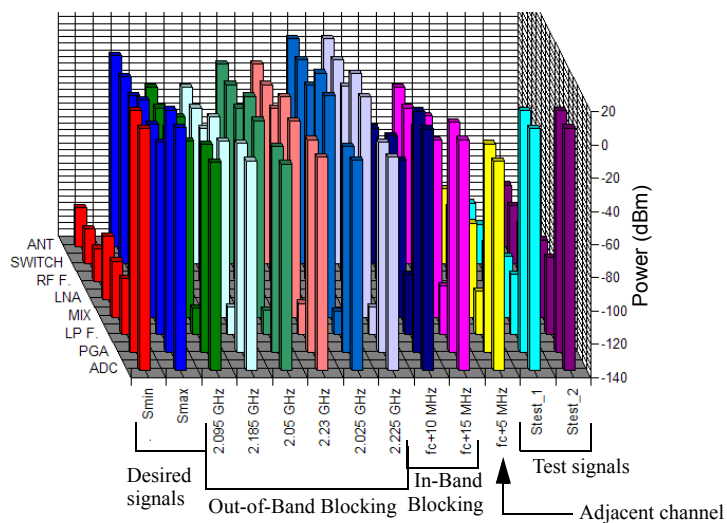

Figure 8. Level diagram for UMTS.

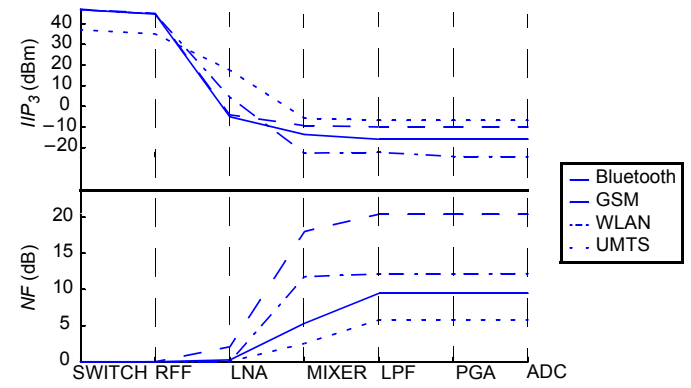

Figure 9. $\mathrm{NF}$ and $\mathrm{IIP}_{3}$ characterization for the different standards.

\section{CONCLUSIONS}

A set of SIMULINK blocks has been proposed for interactive simulation of RF receivers. The building-block models, including main non-ideal effects, have been incorporated in MATLAB by combining SIMULINK library elementary blocks with C-coded S-functions. As an application of the capabilities of the presented toolbox, a multistandard receiver is modeled and simulated to verify the correct performance of the system for GSM, Bluetooth, UMTS and WLAN standards.

\section{REFERENCES}

[1] V. Gazis, N. Alonistioti and L. Merakos:"Toward a Generic Always Best Connected Capability in Integrated WLAN/UMTS Cellular Mobile Networks (and Beyond)". IEEE Wireless Communications, pp. 20-29, June 2005.

[2] M. Brandolini, P. Rossi, D. Manstretta and F. Svelto: "Toward Multistandard Mobile Terminals - Fully Integrated Receivers Requirements and Architectures". IEEE Transactions on Microwave Theory and Techniques, Vol. 53, pp. 1026-1038, March 2005.

[3] G. E. Gielen: "Modeling and analysis techniques for system-level architectural design of telecom front-ends". IEEE Transactions on Microwave Theory and Techniques, Vol. 50, pp. 360-368, Jan. 2002.

[4] J. Crols and M. Steyaert: CMOS Wireless Transceiver Design. Kluwer Academic Publishers, 1997

[5] X. Li and M. Ismail: Multi-Standard CMOS Wireless Design. Kluwer Academic Publishers, 2002

[6] S. Vitali, N. De Laurentiis, G. Albertazzi, F. Agnelli and R. Rovatti: "Multi-Standard Simulation of WLAN/UMTS/GSM Transceivers for Analog Front-End Validation and Design". Proc. of the 2004 IEEE Int. Symposium on Wireless Communication Systems, pp. 20-22, Sept. 2004.

[7] K. Kundert: "Challenges in RF Simulation". Proc. of the 2005 Radio Frequency Integrated Circuits Symposium, pp. 12-14, June 2005.

[8] D. Rodríguez de Llera Gónzalez, A. Rusu, M. Ismail, and H. Tenhunen, "TACT: a multi-standard RF transceiver architecture comparison tool". Proc. of the 48th Midwest Symposium on Circuits and Systems, pp. 659-662, Vol. 1, 2005.

[9] The MathWorks Inc.: "Using Simulink Version 6.0”, 2004.

[10]B. Razavi: RF Microelectronics. Prentice-Hall, 1998.

[11] A.A. Abidi: "Direct-conversion radio transceivers for digital communications”. IEEE J. of Solid-State Circuits, Vol. 30, pp. 1399-1410, Dec. 1995.

[12]A. Savla, A. Ravindran, and M. Ismail: "A reconfigurable low IF-zero IF receiver architecture for multi-standard wide area wireless networks" Proc. IEEE Int. Conf. on Electronics, Circuits and Systems, pp. 935-937, 2003.

[13]H.-K. Yoon and M. Ismail: "A CMOS Multi-standard Receiver Architecture for ISM and UNII Band Applications". Proc. IEEE Int. Symp. Circuits and Systems, pp. IV.265-268, 2004.

TABLE III. SUMMARY OF THE SIMULATED RECEIVER PERFORMANCE

\begin{tabular}{|c|c|c|c|c|c|c|}
\hline \multirow{2}{*}{ Standard } & \multicolumn{2}{|c|}{$N F(\mathbf{d B})$} & \multicolumn{2}{c|}{$\boldsymbol{I P}_{3}(\mathbf{d B m})$} & \multicolumn{2}{c|}{ IIP $_{2}(\mathrm{dBm})$} \\
\cline { 2 - 7 } & Simulated & Specification & Simulated & Specification & Simulated & Specification \\
\hline GSM & 9.48 & 10.00 & -15.96 & -18.00 & 30.39 & 12.50 \\
\hline Bluetooth & 20.42 & 23.00 & -9.93 & -17.50 & 34.48 & 12.00 \\
\hline UMTS & 5.79 & 9.00 & -6.74 & -7.00 & 40.71 & 32.00 \\
\hline WLAN & 12.20 & 15.00 & -24.43 & -24.50 & 5.37 & -4.00 \\
\hline
\end{tabular}

